\title{
Papillary thyroid carcinoma with fibromatosis-like stroma: A case report and review of the literature
}

\author{
ZHIMING WU ${ }^{1}$, XIUFENG CHU $^{1}$, SHENGLONG FAN ${ }^{2}$, XINGCHENG MENG $^{1}$ and CHAOYANG XU ${ }^{3}$ \\ Departments of ${ }^{1}$ General Surgery and ${ }^{2}$ Pathology, Shaoxing Hospital, China Medical University, \\ Shaoxing 312030; ${ }^{3}$ Department of Breast and Thyroid Surgery, Shaoxing People's Hospital, \\ Shaoxing Hospital of Zhejiang University, Shaoxing, Zhejiang 312000, P.R. China
}

Received June 1, 2012; Accepted September 17, 2012

DOI: $10.3892 / 01.2012 .993$

\begin{abstract}
Papillary thyroid carcinoma with fibromatosis-like stroma (PTC-FLS) is a rare type of thyroid carcinoma that does not receive adequate follow-up. This study describes a case of PTC-FLS on the right side of the neck. The patient consented to a right hemithyroidectomy with a central compartment lymphadenectomy. Pathological examination revealed that the two initial lesions were consistent with benign histological performance. Using immunohistology, the proliferated stromal cells were revealed to be positive for vimentin and smooth muscle actin (SMA), but negative for thyroglobulin, S-100 and CK. Post-operative follow-up was scheduled at 3-month intervals for 2 years and then at 6 -month intervals for the next 3 years. There were no signs of tumor recurrence at the 5-year follow-up.
\end{abstract}

\section{Introduction}

Papillary thyroid carcinoma is a common carcinoma. During the past several decades, an increasing incidence of thyroid cancer has been observed in several countries, including China. However, papillary thyroid carcinoma with fibromatosis-like stroma (PTC-FLS) is rare and was first reported in $1832(1,2)$. A discerning characteristic of PTC-FLS is the formation of fibroma (nodular tumor composed of stromal cells) without large growth of papillary carcinoma. The histogenesis and clinicopathological features of PTC-FLS have not yet been fully clarified, due to the rarity of the disease $(3,4)$. Further investigation into PTC-FLS is necessary. In the present study. one particular PTC-FLS case was reported, and the diagnosis and management of this tumor was reviewed through assessment of the literature.

Correspondence to: Dr Chaoyang Xu, Department of Breast and Thyroid Surgery, Shaoxing People's Hospital, The First Affiliated Hospital of Shaoxing University, Shaoxing, Zhejiang 312000, P.R. China

E-mail: xuchaoyang2001@126.com

Key words: carcinoma, stroma, thyroid

\section{Case report}

A 42-year-old female patient was admitted to Shaoxing Hospital (Shaoxing, China), due to a mass on the right side of her neck. This mass had gradually enlarged over the previous 7 months. Upon physical examination, a 3-cm firm nodular mass was revealed in the right lobe of her thyroid gland. Serum free tetra- and tri-iodothyronine (fT4 and fT3) and thyroid stimulating hormone (TSH) tests, in addition to routine laboratory examinations (including thyroid function tests) all exhibited normal results. Additionally, thyroid peroxidase antibody (TPOAb), thyroglobulin antibody ( $\mathrm{Tg} \mathrm{Ab}$ ) and thyroid stimulating hormone receptor antibody (TRAb) were at the normal level. A right hemithyroidectomy with a central compartment lymphadenectomy was performed. Post-operative histologic examination revealed a fibroma-like tumor (Fig. 1). Histological examination identified a complex neoplasm in which a minor component of typical papillary carcinoma was intermingled with a dominant and exuberant stromal proliferation. Immunohistology results revealed that the proliferated stromal cells were positive for vimentin and smooth muscle actin (SMA), but negative for thyroglobulin, S-100 and CK. Post-operative follow-up was scheduled at 3-month intervals for 2 years and then at 6-month intervals for the next 3 years. There were no signs of tumor recurrence at the 5-year follow-up.

The study was approved by the Ethics Committee of Shaoxing Hospital, China Medical University, Shaoxing, China. Written informed consent was obtained from the patient prior to the study.

\section{Discussion}

Here, we presented a case of PTC with fibromatosis-like stroma. Papillary thyroid carcinomas typically contain fibrous stroma of varying degrees and are occasionally almost completely replaced by hyalinized fibrous tissues with multiple or sole microcalcification. However, an extremely large stromal component in PTC is rarely found, which is a typical feature of PTC-FLS (5). Fibromatosis is a tumor-like lesion that develops from fascia or aponeurosis of fibrous tissue. It occurs mainly in the shoulder, chest and back, but rarely in the thyroid. The etiology of PTC-FLS is unknown; 
A

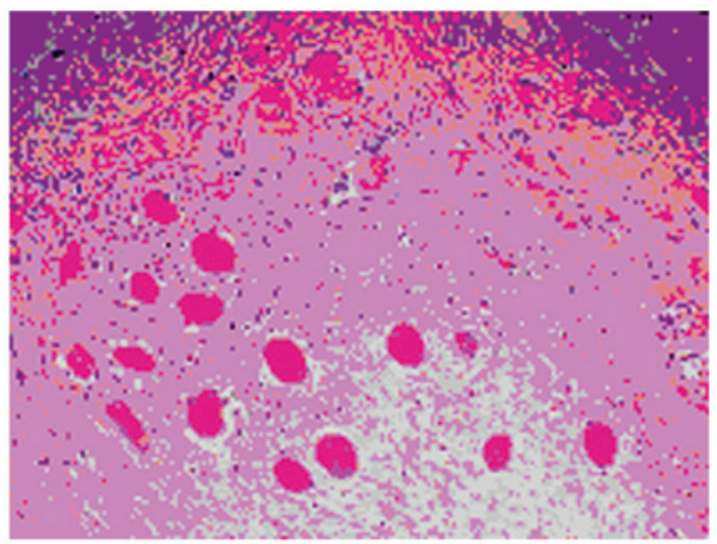

B

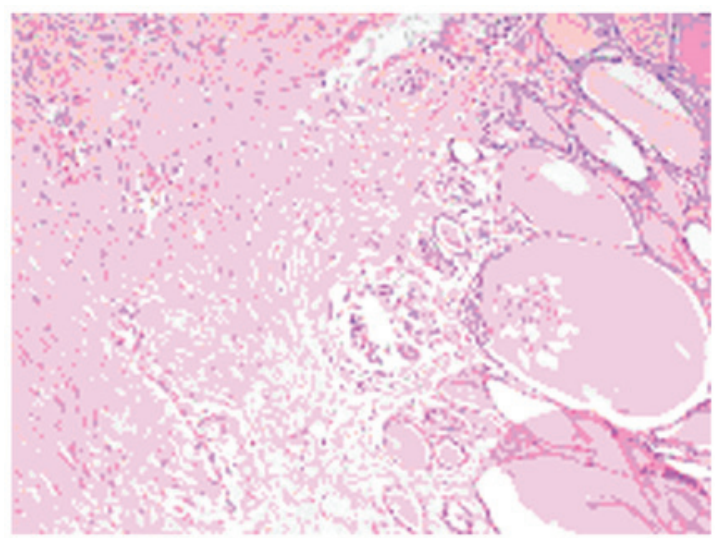

Figure 1. (A) Thin spindle fibroblast infiltration muscle fiber (HE x100) and (B) Spindle-shaped fibroblasts interspersed in the thyroid tissue (HE x100).

in $63 \%$ of cases there is a history of surgical trauma (6). Studies have demonstrated that one possible mechanism is that the stromal component is an exaggerated mesenchymal reaction to injuries resulting from tumor invasion into the stroma. Another possibility is that the stromal component is hormone dependented tumorigenesis in nature (7). Notably, it has been revealed that estrogen receptors are involved in $30 \%$ of PTC-FLS cases (8). Proliferating fibroblasts that had formed lumps lined with carcinomatous epithelial components were observed, which were similar to the leaf-like structures identified in breast phyllodes tumors. PTC-FLS is considered to be at high-risk of misdiagnosis, due to its rarity and fast-growing biological behavior, in the same way as other malignant tumors.

On palpation, thyroid fibromatosis may be confused with thyroid cancer; computerized tomography imaging revealed the fibromatosis to be a large, irregular mass with a lobulated edge and no capsule, exhibiting invasive growth into soft tissues and surrounding muscles. Therefore, this mass may be easily misdiagnosed as a malignant tumor (7). Although contrast-enhanced CT scans reveal the rich blood supply in thyroid fibromatosis, this is ineffective in differentiating thyroid fibromatosis from thyroid cancer. The definitive diagnosis of PTC-FLS may be determined by examining an intra-operative frozen section and a post-operative paraffin section. PTC-FLS must be treated surgically as a low-grade malignant tumor. As the recurrence rate for local excision is $20-45 \%$, complete excision needs to be performed (9). In certain cases, when tumors invade nerves and significant organs, it is difficult to perform this procedure. Invaded organs have a greater impact on the patient's quality of life, rendering complete surgery a contradictory solution. However, we suggest that complete surgery should be performed if possible.

For patients who do not wish to undergo surgery or cannot afford the operation, local excision in addition to radiation therapy may be an effective treatment. Radiation therapy is more suitable, as the recurrence rate is reduced with a radiation dose of 50-60 Gy (10). However, there are also numerous studies demonstrating the ineffectiveness of radiotherapy (10). A number of systemic therapies have been studied for inoper- able cases of fibromatosis, such as antiestrogens; nonsteroidal anti-inflammatory drugs (NSAIDs), including indomethacin, sulindac and cyclo-oxygenase-2 (COX-2) inhibitors; interferons and various other drugs, including methotrexate in combination with doxorubicin, ifosfamide and vinblastine or vinorelbine. In addition, more recently, target therapy with imatinib has been applied in platelet-derived growth factor receptor-beta (PDGFR- $\beta$ )-positive tumors. Chemotherapy with a low-dose of methotrexate and vinblastine (or its less neurotoxic analog, vinorelbine) on a weekly basis has been demonstrated to achieve a $70 \%$ relapse-free survival rate at 10 years (11). Likewise, indomethacin has been demonstrated to be effective in certain cases of fibromatosis (12). Traditional Chinese medicine may also be effective in this disease.

The prognosis of this tumor depends on its size and location. Typically, the 10 -year survival rate is $94 \%$ and the 20 -year survival rate is $86 \%$ (11). However, for patients with aggressive fibromatosis involving vital organs or large blood vessels, the survival rate is lower.

\section{References}

1. Mizukami Y, Nonomura A, Matsubara F, Michigishi T, Ohmura K and Hashimoto T: Papillary carcinoma of the thyroid gland with fibromatosis-like stroma. Histopathology 20: 355-357, 1992.

2. Mizukami Y, Kurumaya H, Kitagawa T, Minato H, Nonomura A, Michigishi T and Noguchi M: Papillary carcinoma of the thyroid gland with fibromatosis-like stroma: a case report and review of the literature. Mod Pathol 8: 366-370, 1995.

3. Arena S, Salamone S, Cianci R, Scollo C, Masucci R, Giannone G, Manusia M, Vigneri R and La Rosa GL: Aggressive fibromatosis of the neck initiated after thyroidectomy. J Endocrinol Invest 29: 78-81, 2006.

4. Schwarzlmüller B and Hofstädter F: Fibromatosis of the thyroid gland region. An electron-microscopic and enzyme-histochemical study. Virchows Arch A Pathol Anat Histol 377: 145-155, 1978 (In German).

5. Shields CJ, Winter DC, Kirwan WO and Redmond HP: Desmoid tumours. Eur J Surg Oncol 27: 701-706, 2001.

6. Pepe P, Panella P, Pennisi M and Aragona F: Does color Doppler sonography improve the clinical assessment of patients with acute scrotum? Eur J Radiol 60: 120-124, 2006.

7. Russ G, Bigorgne C, Royer B, Rouxel A and Bienvenu Perrard M: The thyroid imaging reporting and data system (TIRADS) for ultrasound of the thyroid. J Radiol 92: 701-713, 2011 (In French). 
8. Mizukami Y, Nonomura A, Matsubara F, Michigishi T, Ohmura $\mathrm{K}$ and Hashimoto T: Papillary carcinoma of the thyroid gland with fibromatosis-like stroma. Histopathology 20: 355-357, 1992.

9. Clark SK and Phillips RK: Desmoids in familial adenomatous polyposis. Br J Surg 83: 1494-1504, 1996.

10. Kiel KD and Suit HD: Radiation therapy in the treatment of aggressive fibromatoses (desmoid tumors). Cancer 54: 2051-2055, 1984.
11. Ballo MT, Zagars GK, Pollack A, et al: Desmoid tumor: prognostic factors and outcome after surgery, radiation therapy, or combined surgery and radiation therapy. J Clin Oncol 7: 158-167, 1999.

12. Easter DW and Halasz NA: Recent trends in the management of desmoid tumors. Ann Surg 210: 765-769, 1989. 